\section{Methods and media}

\section{OP46 DEVELOPMENT AND VALIDATION OF A SENSITIVE SEARCH FILTER TO IDENTIFY EQUITY-FOCUSED STUDIES}

SL Prady*, EP Uphoff, M Power, S Golder. Department of Health Sciences, University of York, York, UK

\subsection{6/jech-2018-SSMabstracts.46}

Background Health inequalities, worse health associated with social and economic disadvantage, are reported by a minority of research articles. For example, fewer than 15\% of intervention studies report any outcome by a social determinant of health. Systematic reviewers wishing to conduct an equity review are hampered in identifying these studies by a deficit in standardised terminology, uncertainties around indexing and an absence of validated search filters. Current reporting guidelines recommend not to apply equity filters. The resulting manual effort needed to screen for reports of equity can be considerable. We aimed to design and test the sensitivity of search filters to locate studies that reported outcomes by a social determinant of health.

Methods We developed and expanded a 'specific terms strategy' using keywords and subject headings compiled from systematic reviews that applied an equity filter. A 'non-specific strategy' was compiled from phrases used to describe equity analyses reported in titles and abstracts, and related subject headings. Gold standard papers were independently collated using three different methods and randomly split into evaluation and (internal) validation sets. We set a target of 0.90 sensitivity $(95 \%$ binomial confidence interval; 0.84, 0.94) in retrieving 150 gold standard validation papers. The filters were developed in MEDLINE, adapted for Embase and tested in both. We noted the reduction in the number needed to screen in a proposed equity-focused systematic review and the proportion of equity-focused systematic reviews (published in 2014-2017) we assessed in the project that applied an equity filter to their search strategy.

Results The specific terms strategy filtered out 93\%-95\% of all records, and had a sensitivity for retrieving the validation set of articles 0.84 in MEDLINE (0.77, 0.89), and 0.87 (0.81, 0.92) in Embase. When combined (Boolean 'OR') with the non-specific strategy, sensitivity was $0.92(0.86,0.96)$ in MEDLINE and $0.94(0.89,0.97)$ in Embase. After applying the specific terms strategy the number needed to screen was reduced by $77 \%$, and by $59.7 \%$ (MEDLINE) and $63.5 \%$ (Embase) after applying the combined strategy. Of the 128 reviews we assessed in the project, $83 \%$ applied an equity filter to their search strategy.

Conclusion A combined approach of specific and non-specific terms is recommended if systematic reviewers wish to filter studies for reporting outcomes by social determinants. Our filters are lengthy and focus on sensitivity. Future research should examine specificity and validate using external sources. Indexing standardisation improvement is needed for equity studies.
OP47 TRANSPARENCY IN THE REPORTING AND CONDUCT OF NARRATIVE SYNTHESIS OF QUANTITATIVE DATA IN SYSTEMATIC REVIEWS

${ }^{1} \mathrm{M}$ Campbell ${ }^{*},{ }^{1} \mathrm{SV}$ Katikireddi, ${ }^{2} \mathrm{~A}$ Sowden, ${ }^{3} \mathrm{JE}$ McKenzie, ${ }^{1} \mathrm{H}$ Thomson. ${ }^{1} \mathrm{MRC/CSO}$ Social and Public Health Sciences Unit, University of Glasgow, Glasgow, UK; ${ }^{2}$ Centre for Reviews and Dissemination, University of York, York, UK; ${ }^{3}$ School of Public Health and Preventive Medicine, Monash University, Melbourne, Australia

\subsection{6/jech-2018-SSMabstracts.47}

Background Well conducted systematic reviews provide transparent and robust evidence syntheses. They are, therefore, important for informing clinical practice and health policy. In systematic reviews where meta-analysis is not possible or appropriate, quantitative data are often synthesised narratively. However, a major concern is that narrative synthesis (NS) can lack transparency, leading to bias in the synthesis and threatening the reliability of these reviews for decision makers. We conducted a systematic assessment of Cochrane reviews to establish prevalence of NS, and to assess current practice and transparency in the conduct and reporting of NS in systematic reviews.

Methods Cochrane systematic reviews published between April 2016 and April $2017(n=714)$ were examined to determine the primary method of synthesis. Data were extracted from a sample of reviews $(n=60 / 714)$ that used NS as the primary method of synthesis. A previously tested data extraction template, based on key guidance on NS, was used. This covered reporting of NS methods; transparency between data and text; management and investigation of heterogeneity; and review authors' reflections on limitations of the synthesis.

Results NS or text only as the primary method of synthesis was used by $16 \%(n=113 / 714)$. In reviews using NS $(n=60 /$ 113): $53 \%$ stated the data were being synthesised narratively; $18 \%$ described the NS methods; and 10\% referred to NS guidance. Links between the text and the data were clear in $30 \%(18 / 60)$ and, partially in $23 \%$ of reviews. The remaining $47 \%$ of reviews did not present tabulated summaries of synthesised data. Of the reviews that provided tabulated summaries of data, $16 \%(n=5 / 32)$ did not present the data in the same order and categories as the narrative text. Heterogeneity in the direction of effect of the primary outcome was identified in 14 reviews, of these $46 \% \quad(n=6 / 14)$ attempted to explain the heterogeneity. While $98 \%$ of review authors reflected on the limitations of the evidence, $77 \%$ reported limitations of the synthesis process.

Conclusion Despite being commonly used, NS is not reported transparently. The limited reporting of methods, and lack of transparency between the data, the narrative and the conclusions, limits assessment of the validity and threatens the reliability of systematic reviews using NS. There is a need for updated guidance on NS. A Delphi survey is underway to develop consensus-based a reporting guideline for NS. The draft items arising from the Delphi survey will be presented. 